\title{
Understanding large SEP events with the PATH code: Modeling of the 13 December 2006 SEP event
}

\author{
O. P. Verkhoglyadova, ${ }^{1}$ G. Li, ${ }^{1,2}$ G. P. Zank, ${ }^{1,2}$ Q. Hu, ${ }^{1}$ C. M. S. Cohen, ${ }^{3}$ R. A. Mewaldt, ${ }^{3}$ \\ G. M. Mason, ${ }^{4}$ D. K. Haggerty, ${ }^{4}$ T. T. von Rosenvinge, ${ }^{5}$ and M. D. Looper ${ }^{6}$ \\ Received 28 April 2010; revised 27 September 2010; accepted 7 October 2010; published 7 December 2010.
}

[1] The Particle Acceleration and Transport in the Heliosphere (PATH) numerical code was developed to understand solar energetic particle (SEP) events in the near-Earth environment. We discuss simulation results for the 13 December 2006 SEP event. The PATH code includes modeling a background solar wind through which a CME-driven oblique shock propagates. The code incorporates a mixed population of both flare and shock-accelerated solar wind suprathermal particles. The shock parameters derived from ACE measurements at $1 \mathrm{AU}$ and observational flare characteristics are used as input into the numerical model. We assume that the diffusive shock acceleration mechanism is responsible for particle energization. We model the subsequent transport of particles originated at the flare site and particles escaping from the shock and propagating in the equatorial plane through the interplanetary medium. We derive spectra for protons, oxygen, and iron ions, together with their time-intensity profiles at 1 AU. Our modeling results show reasonable agreement with in situ measurements by ACE, STEREO, GOES, and SAMPEX for this event. We numerically estimate the $\mathrm{Fe} / \mathrm{O}$ abundance ratio and discuss the physics underlying a mixed SEP event. We point out that the flare population is as important as shock geometry changes during shock propagation for modeling time-intensity profiles and spectra at $1 \mathrm{AU}$. The combined effects of seed population and shock geometry will be examined in the framework of an extended PATH code in future modeling efforts.

Citation: Verkhoglyadova, O. P., G. Li, G. P. Zank, Q. Hu, C. M. S. Cohen, R. A. Mewaldt, G. M. Mason, D. K. Haggerty, T. T. von Rosenvinge, and M. D. Looper (2010), Understanding large SEP events with the PATH code: Modeling of the 13 December 2006 SEP event, J. Geophys. Res., 115, A12103, doi:10.1029/2010JA015615.

\section{Introduction}

[2] Large solar energetic particle events (SEPs) attract attention because of their high energy releases $(\sim 10 \mathrm{GeV}$ for protons), their evident importance for increasing the nearEarth radiation environment [Feynman and Gabriel, 2000; Tylka et al., 2005; Desai et al., 2006], and the corresponding effects on the Earth's magnetosphere and ionosphere [Tsurutani et al., 2009]. Considerable progress has been achieved recently in modeling particle acceleration and transport in SEP events (see the review by Zank et al. [2007] and references therein). More studies are in progress including detailed descriptions of the initial stages of CME shock formation [Manchester et al., 2005, 2008; Zhang and Wu,

\footnotetext{
${ }^{1}$ Center for Space Plasma and Aeronomic Research, University of Alabama in Huntsville, Huntsville, Alabama, USA.

${ }^{2}$ Department of Physics, University of Alabama in Huntsville, Huntsville, Alabama, USA.

${ }^{3}$ California Institute of Technology, Pasadena, California, USA.

${ }^{4}$ Applied Physics Lab., Johns Hopkins University, Laurel, Maryland, USA.

${ }^{5}$ NASA Goddard SFC, Greenbelt, Maryland, USA.

${ }^{6}$ The Aerospace Corporation, Los Angeles, California, USA.

Copyright 2010 by the American Geophysical Union. 0148-0227/10/2010JA015615
}

2009], modeling of a shock dynamics and connection to an observer at 1 AU [Luhmann et al., 2007, 2010], more detailed wave turbulence model at an oblique shock, the observed variability of particle composition and the variability and evolution of spectra at $1 \mathrm{AU}$ [Tylka et al., 2005; Tylka and Lee, 2006]. The latter problem has been studied both theoretically and numerically in a series of papers [Zank et al., 2000; Rice et al., 2003; Li et al., 2003, 2005; Zank et al., 2007; Verkhoglyadova et al., 2009]. These authors have developed a comprehensive numerical model, called Particle Acceleration and Transport in the Heliosphere (PATH), to study the variability of particle spectra and intensities at 1 AU during a SEP event.

[3] It has been suggested [Cane et al., 1986; Reames, 1999] that SEP events can be divided coarsely into two categories, impulsive and gradual events. In the former, energetic particles are accelerated at a flare site and travel along the Parker spiral magnetic field lines to reach the Earth, with the particle intensity profiles exhibiting a rapid rise followed by a relatively rapid decaying phase. Gradual events are believed to be manifestations of particles accelerated at a CME-driven shock which are accelerated and escape over its lifetime, and are observed as elevated particle intensities at the Earth's orbit assuming magnetic connectivity between the acceleration site 


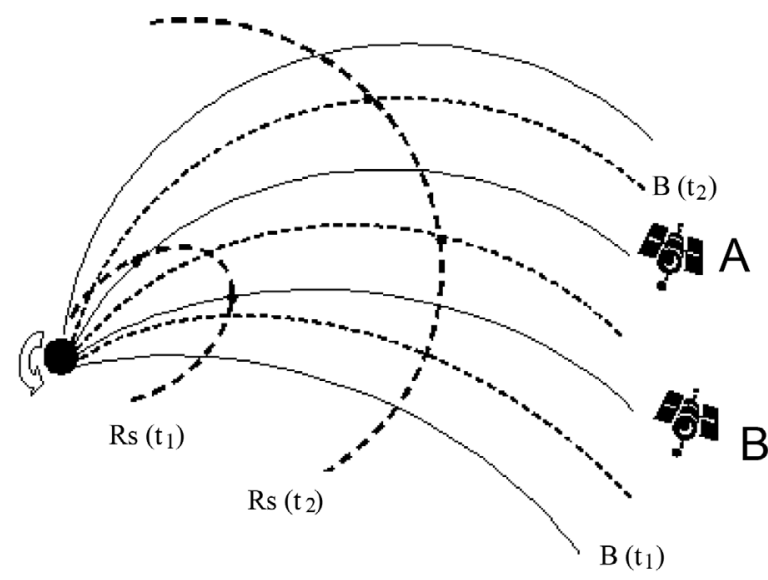

Figure 1. Schematic illustrating shock angle observations. IMF lines are shown by solid and dashed lines, corresponding to $t_{1}$ and $t_{2}$, where $t_{1}<t_{2}$. Consecutive shock positions are indicated by $\operatorname{Rs}\left(\mathrm{t}_{1}\right)$ and $\mathrm{Rs}\left(\mathrm{t}_{2}\right)$. We discuss observations made by two spacecraft $\mathrm{A}$ and $\mathrm{B}$.

at the shock and the Earth. Particle composition and charge states for these two types of SEP events are quite different. For instance, impulsive events manifest themselves in a higher abundance of heavy ions relative to oxygen (sometimes described as "iron-rich") and enhancements of the isotopic ratio of ${ }^{3} \mathrm{He} /{ }^{4} \mathrm{He}$ [Reames, 1999; Kallenrode, 2003].

[4] These two cases of particle acceleration exhibit different temporal dynamics of particle intensities at $1 \mathrm{AU}$, also depending on magnetic connection between the flare site on the Sun and the Earth. Impulsive events manifest themselves in an abrupt rise of the most energetic particles around the flare onset time. Gradual events can show an increase in lowenergy particles at $1 \mathrm{AU}$ around the CME-driven shock arrival time. The latter increase is often called an ESP (energetic storm particle) event. However, some of the gradual events associated with a source magnetically connected to the Earth show sharp rises in particle intensities at the SEP event onset and have a composition that is similar to that of impulsive events [Cohen et al., 1999]. It has been suggested that pure impulsive and pure gradual events are the limiting cases of SEP events and there is a continuous transition between different kinds of SEP events [Kallenrode, 2003]. It should be noted that spectra of flare particles (if present) as well as interplanetary propagation effects can play a role in determining fractional content $(\mathrm{Fe} / \mathrm{O}$ abundance ratio). We will discuss these issues in section 5 .

[5] SEP events that exhibit distinct signatures of both impulsive and gradual events are often regarded as "mixed" events. These SEP events may show two peaks in their timeintensity profiles [Cane et al., 2003]. One explanation for a mixed event is that it is a mixture of flare-accelerated and shock-accelerated particles, i.e., two distinct acceleration mechanisms are involved [Cane et al., 2003, 2006]. Thus, the higher-energy peak in the particle intensity is caused by flare particles and the lower-energy peak is caused by shockaccelerated particles. Li and Zank [2005] have modeled timeintensity profiles for a mixed event. They assumed that flare particles are accelerated at the flare site, but a low-energy portion of them can be absorbed by the shock in which case they could undergo acceleration at the shock and become shock-accelerated particles. At a later time, as the shock propagates into the solar wind and weakens, it becomes less efficient in accelerating and trapping flare particles. A portion of the escaping flare particles can also reverse their direction and come back to the shock due to scattering in the solar wind, depending on the particle pitch angles. Those particles that satisfy the resonance condition with waves in the shock vicinity (see Li et al. [2003, 2005] for details) can be reaccelerated by the shock. Throughout the event the shock is transparent to higher-energy flare particles, which come directly from the flare site to 1 AU. Modeled time-intensity profiles for a mixed event [ $L i$ and Zank, 2005] plotted for different particle energies show initial peaks due to flare particles which were not reprocessed by the shock and gradual increases at later times caused by shock-accelerated particles (including a portion of flare particles absorbed and reaccelerated by the shock). This approach assumes two distinct particle populations contributing to the energetic particle intensities through different acceleration mechanisms (on-site acceleration at a flare site and acceleration at an evolving CME-driven shock), where the differing fractional content of the seed particle population results in a more impulsive-like or more gradual-like type of SEP event [Kallenrode, 2003].

[6] A different point of view to explain temporal variability of particle spectra and intensities is through the evolution of the shock geometry (shock angle or obliquity) as the shock propagates [Tylka et al., 2005; Tylka and Lee, 2006; Sandroos and Vainio, 2007]. The concept is illustrated in Figure 1. The CME-driven shock locations at times of $t_{1}$ and $t_{2}$ are indicated by $\operatorname{Rs}\left(t_{1}\right)$ and $R s\left(t_{2}\right)$, respectively. Interplanetary magnetic field (IMF) lines at $t_{1}, B\left(t_{1}\right)$, are shown by solid lines. Due to the Sun's rotation, positions of the same field lines change to $\mathrm{B}\left(\mathrm{t}_{2}\right)$ at $\mathrm{t}_{2}$ (shown by dashed lines). We trace the shock geometry (shock angles) at consecutive locations along the shock front at $t_{1}$ and $t_{2}$ (shown by black dots), which are magnetically connected to the spacecraft A and $\mathrm{B}$. At $\mathrm{t}_{1}$, spacecraft $\mathrm{A}$ is connected by the IMF to a quasiperpendicular region of the CME-driven shock located at $\operatorname{Rs}\left(\mathrm{t}_{1}\right)$ and observes particles accelerated locally at a quasiperpendicular shock. At the same time, spacecraft B is connected to a quasiparallel shock region and observes particles accelerated locally at a quasiparallel shock. At a later time $t_{2}$, spacecraft $A$ is connected to a quasiparallel region of the shock located at $\operatorname{Rs}\left(\mathrm{t}_{2}\right)$, and spacecraft $\mathrm{B}$ is still connected to a quasiparallel region. In this case, spacecraft A observes a CME-driven shock geometry changing from a quasiperpendicular (near the Sun) to a quasiparallel (at $1 \mathrm{AU}$ ) configuration. Because quasiperpendicular shocks require higher injection thresholds and generally result in harder spectra, this geometry change may provide for efficient acceleration of a suprathermal seed population in the higherenergy part of the particle spectra at the beginning of the SEP event. This early phase is followed by a later phase with more efficient acceleration in a lower-energy part of the spectra due to a quasiparallel shock. Note that spacecraft B does not observe the shock geometry change, which motivates our modeling with the assumption of a continually quasiparallel interplanetary shock. Additionally, spacecraft at 1 AU are connected to different field lines at different times. Second, if the speed of energetic particles can be taken as infinity (these particles are much faster than the solar wind speed of 
$\sim 400 \mathrm{~km} / \mathrm{s}$ ), then they will move from the source, which is along the shock surface, to the observer instantaneously. In reality, finite particle speeds as well as pitch angle scattering means that particle accelerated at different times (therefore different part of the shock) will reach the spacecraft at the same time. We should note that a comprehensive picture of an observer-shock connection and evolution of particle intensities at $1 \mathrm{AU}$ requires more adequate modeling (see for instance Luhmann et al. [2007]).

[7] To predict energetic particle spectra and intensities at 1 AU, we model a specific SEP event with the PATH code. In this paper, we do not simulate the changing geometry of a propagating shock. Thus we cannot compare directly the above two possibilities for describing a mixed SEP event. The focus of this paper is to study the validity of the approach that assumes a mixed seed particle population. An indirect comparison with the alternative description will be made and both possibilities will be discussed in section 5 .

[8] In this paper, we focus on a large SEP event that occurred on 13 December 2006. This event began at 0214:00 UT on 13 December with an X3.4 flare (active region located around $\sim \mathrm{W} 25$ longitude) of $43 \mathrm{~min}$ duration and reached its peak intensity at $\sim 0240: 00$ UT (seen in $\mathrm{X}$-ray fluxes measured by GOES-12; Figure 2, first panel). The eruption of a halo CME was first observed around $\sim 0254: 04$ UT (as indicated in the SOHO/LASCO CME Catalog, courtesy of the CDAW Data Center, GSFC) and could be associated with this flare. The flare was accompanied by a substantial increase in energetic particles observed at the Earth's orbit. Flare particles may have contributed to an almost immediate rise in energetic proton intensities above $1 \mathrm{MeV}$ according to GOES-12 satellite measurements (Figure 2, second panel) and neutron monitor measurements (Figure 2, fourth panel). A CME-associated shock reached $1 \mathrm{AU}$ within about $35 \mathrm{~h}$ (seen in GOES magnetic field measurements in Figure 2, third panel) and caused an ESP event seen in protons above $1 \mathrm{MeV}$ (Figure 2, second panel). The paper by Liu et al. [2008] provides a multisatellite view of this SEP event. From the observations shown in Figure 2 we suggest this SEP event as a possible example of a mixed event. Observations of element abundances by ACE and STEREO for this event can also be interpreted as indicative of a mixed particle population (see Cohen et al. [2008a] and section 5). They show that eventintegrated abundances (relative to oxygen) are close to those for an impulsive event. However, the isotopic ratio ${ }^{3} \mathrm{He} /{ }^{4} \mathrm{He}$ is not large and is close to that measured in a gradual event that occurred several days before.

[9] In this paper we extend our PATH code to model the mixed event of 13 December 2006, to understand the details of solar wind particle acceleration at an evolving shock and to estimate energetic particle intensities and spectra at $1 \mathrm{AU}$ [see also Verkhoglyadova et al., 2008; Tsurutani et al., 2009]. Details of the model are presented next.

\section{Model}

[10] Our numerical model of an SEP event incorporates realistic boundary conditions close to the Sun, modeling of the solar wind background, and propagation of a CME-driven evolving shock throughout the inner heliosphere. Another part (or block) of the code include particle injection into the moving shock, particle diffusion and acceleration at the shock vicinity, and further transport to several AU. In this paper we apply the PATH code to study the temporal evolution of the near-Earth energetic particle population in an SEP event. It is based on the original "onion-shell" model approach proposed by Zank et al. [2000] and further developed in a number of papers [Rice et al., 2003; Li et al., 2003, 2005]. The core of the model is particle acceleration on MHD wave turbulence in the vicinity of a quasiparallel shock, i.e., diffusive shock acceleration. By scattering on the MHD turbulence, particles return to and cross the shock repeatedly, gaining energy in each cycle. Rice et al. [2003] adapted a self-consistent approach of Lee [1983] and Gordon et al. [1999], which introduced Alfvén wave excitation at a quasiparallel shock through wave-particle interaction with streaming protons and estimated parallel diffusion coefficient for those particles, extending the original Zank et al. [2000] treatment of selfconsistently coupled wave excitation. The PATH model solves the kinetic transport equation for energetic particles in the immediate vicinity of the shock. At the shock, the accelerated particle spectrum is approximated by a solution for a planar shock, and the time-dependent shock parameters (i.e., the shock Mach number and compression ratio) are regarded as locally constant. An instantaneous accelerated particle distribution $(f)$ at the shock is a power law: $f \sim p^{-\beta}, \quad \beta=\frac{3 s_{i}}{s_{i}-1}, \quad$ where $s_{i}$ is the instantaneous shock compression ratio at the $\mathrm{i}^{\text {th }}$ step of the code. Particles escaping from the shock complex are followed in the transport module of the code using a Monte Carlo approach. Particles move along interplanetary magnetic field lines while experiencing scattering in pitch angle due to interaction with interplanetary turbulence. This effect is modeled with a mean free path $(\lambda)$ which is a function of particle momentum $(p)$, heliocentric distance $(r)$ and a mass to charge ratio $(A / Q)[\mathrm{Li}$ et al., 2005]:

$$
\lambda=0.8 A U\left(\frac{p c}{1 G e V}\right)^{1 / 3}\left(\frac{A}{Q}\right)^{1 / 3}\left(\frac{r}{1 A U}\right)^{2 / 3}
$$

[11] Resultant particle spectra observed at 1 AU are a function of superposed different instantaneous accelerated spectra and transport effects. The physical and numerical background of the PATH model has been recently reviewed by Zank et al. [2007]. It has been shown that the model can be applied to reconstruct realistic energetic particle spectra at $1 \mathrm{AU}$ in a gradual SEP event associated with a quasiparallel CME-driven shock [Verkhoglyadova et al., 2009]. In the current study we extend the code to model a mixed SEP event at an oblique shock and make detailed comparison with in situ satellite measurements. Below we list the main modifications made to the code as compared to the previous result [Verkhoglyadova et al., 2009].

[12] Following the approach by Li and Zank [2005] the PATH code has been modified to include a contribution from flare particles, which were assumed to have a power law spectrum and maximum energy of $1 \mathrm{GeV}$ (see section 4 for details). A flare duration of $43 \mathrm{~min}$ is taken from GOES observations for the 13 December 2006 event. High-energy flare particles are assumed to reach $1 \mathrm{AU}$ without interacting with the shock. A portion of lower-energy flare particles will be absorbed and possibly reaccelerated by the shock. At any 


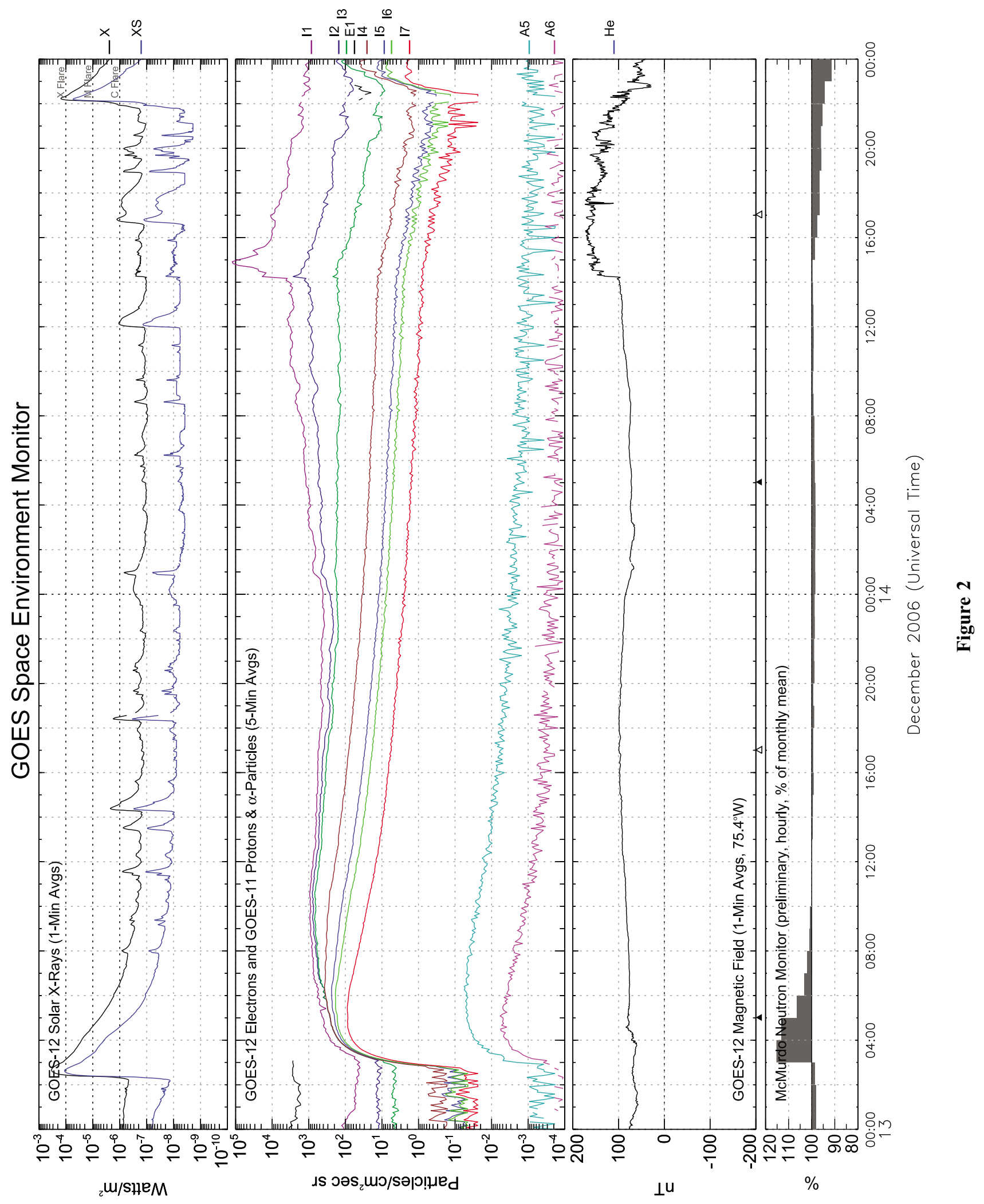




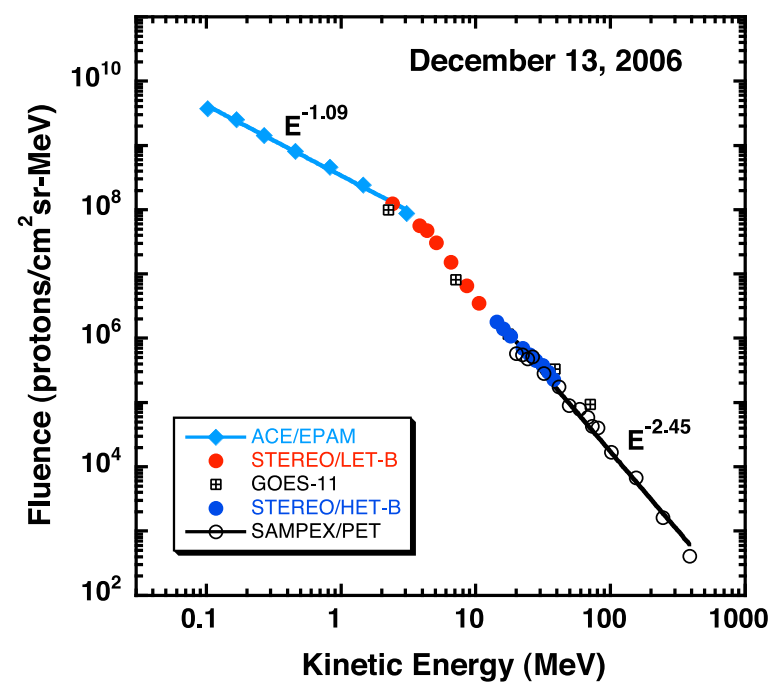

Figure 3. Event-integrated energetic proton spectra obtained with ACE, STEREO, GOES-11, and SAMPEX for the 13 December 2006 SEP event.

time, the maximum energy for a particle to interact with the shock is assumed to be the maximum achievable energy at the shock, which is given by the resonance conditions. Above this energy, we assume the particles can penetrate the shock and do not interact with the shock. Note that this assumption is crude because the turbulence in the downstream is stronger than that in the upstream. Furthermore, compression at the shock leads to a higher wave number. More detailed simulation should consider particle and wave interaction in the downstream, which is beyond the scope of this work. Since the limiting energy changes with time as the shock evolves, an important feature of the PATH code is the incorporation of flare particles into the time-dependent injection, trapping and acceleration process in the shock vicinity that contributes to the total SEP spectrum.

[13] Another modification to the PATH code is the inclusion of perpendicular diffusion for an oblique shock. We followed the approach of Matthaeus et al. [2003] and Zank et al. [2004, 2006] who developed a Nonlinear Guiding Center theory (NLGC) for the perpendicular diffusion coefficient $\left(\kappa_{\perp}\right)$. Thus, the particle diffusion coefficient at the shock can be estimated as: $\kappa=\kappa_{\|} \cos ^{2} \theta+\kappa_{\perp} \sin ^{2} \theta$, where $\kappa$, $\kappa_{\|}$and $\theta$ are the total diffusion coefficient, the parallel diffusion coefficient, and the angle between the shock normal and background magnetic field, respectively. We assumed that the shock has the same obliquity as estimated from ACE observations at $1 \mathrm{AU}$, i.e., $\theta \sim 30^{\circ}$ (see also section 4 ). We believe that the reasonably good agreement between our modeling results and observations (see section 4) favors this simplification of the model (see also Figure 1 for spacecraft B). A different approach explains observed temporal evolution of particle intensities at $1 \mathrm{AU}$ by changes in the shock geometry from a quasiperpendicular configuration to a quasi-parallel configuration during its propagation (see section 1). Such a geometry change would be observed by spacecraft $\mathrm{A}$ where its magnetic connection to different regions of an evolving shock is shown in Figure 1. We will return to this approach in section 5. Here, using the modified PATH modeling approach, we study time intensity profiles and particle spectra for both protons and heavy ions (Fe and $\mathrm{O}$ ) at $1 \mathrm{AU}$ and compare these results with observations. Section 3 presents some of the relevant in situ observations of the event we model.

\section{ACE, STEREO, GOES, and SAMPEX Observations of the 13 December 2006 SEP Event at $1 \mathrm{AU}$}

\subsection{Particle Spectra at 1 AU}

[14] First measurements of the proton fluence spectrum for the 13 December 2006 event were presented by Mewald et al. [2008a] and Cohen et al. [2008a]. Figure 3 shows a composite proton spectrum over a large energy range (from $100 \mathrm{keV}$ to $\sim 400 \mathrm{MeV}$ ) integrated over the entire event and based on observations by ACE, STEREO, GOES-11 and SAMPEX. To a very good agreement, the SAMPEX/PET and STEREO/HET-B data can be fitted by a power law. The GOES-11 data is also consistent with the fitted power law. The entire spectrum shows two distinct power law slopes, for lower- and higher-energy regions, and a transition region (spectral break) in between. Any model attempting to describe this event must be capable of reproducing the double power law for the proton event-integrated spectrum.

\subsection{Time-Intensity Profiles at $1 \mathbf{A U}$}

[15] Using ACE and STEREO measurements in both lowand high-energy ranges, we examine the time-intensity profiles of the protons and oxygen ions. In Figures 4-7 we present observations (top) and modeling results (bottom) for comparison purposes. Only three energy channels are shown on each plot. Figure 4 (top) shows protons measured by the HET instrument on STEREO B [von Rosenvinge et al., 2008], illustrating the major peak after the flare start (see also Figure 2 by von Rosenvinge et al. [2009]). The proton intensity at $\sim 20 \mathrm{MeV}$ decreases steeply with time, whereas protons with energies $12-17 \mathrm{MeV}$ show only a small decrease up to the approximate shock arrival time which corresponds to the right-hand end of the panel. Low-energy particles (below $3 \mathrm{MeV}$ ) are studied using the ACE/ULEIS instrument [Mason et al., 1998]. Observed proton intensities at $\sim 160 \mathrm{keV}-2.56 \mathrm{MeV}$ start rising after a larger delay from the

Figure 2. GOES Space Environment Monitor observations at 1 AU during the SEP event (courtesy of NOAA National Geophysical Data Center). (first panel) The X-ray flare onset at 0400 UT on 13 December. (second panel) Energetic proton intensities in the range from $1 \mathrm{MeV}$ to $100 \mathrm{MeV}$ (indicated by "I," energy increases from top to bottom). (third panel) Interplanetary magnetic field with the CME-driven shock arrival time at 1 AU (around 1400 UT on 14 December). (fourth panel) Cosmic ray background increases. The first panel shows the whole Sun X-ray fluxes in two wavelength bands: XL 1-8 A X-rays (Watts/ $\mathrm{m}^{2}$ ) and XS 0.5-3 $\AA$ X-rays (Watts $/ \mathrm{m}^{2}$ ). The second panel shows seven ion energy channels: $\mathrm{I} 1>1 \mathrm{MeV}\left(\mathrm{protons} / \mathrm{cm}^{2} \mathrm{~s} \mathrm{sr}\right)$, $\mathrm{I} 2>5 \mathrm{MeV}$ (protons $/ \mathrm{cm}^{2} \mathrm{~s} \mathrm{sr}$ ), I3 $>10 \mathrm{MeV}$ (protons $/ \mathrm{cm}^{2} \mathrm{~s} \mathrm{sr}$ ), I4 $>30 \mathrm{MeV}$ (protons $/ \mathrm{cm}^{2} \mathrm{~s} \mathrm{sr}$ ), I5 $>50 \mathrm{MeV}$ ( $\mathrm{protons} / \mathrm{cm}^{2} \mathrm{~s} \mathrm{sr}$ ), I6 $>60 \mathrm{MeV}$ (protons $/ \mathrm{cm}^{2} \mathrm{~s} \mathrm{sr}$ ), and I7 $>100 \mathrm{MeV}$ (protons $/ \mathrm{cm}^{2} \mathrm{~s} \mathrm{sr}$ ). 

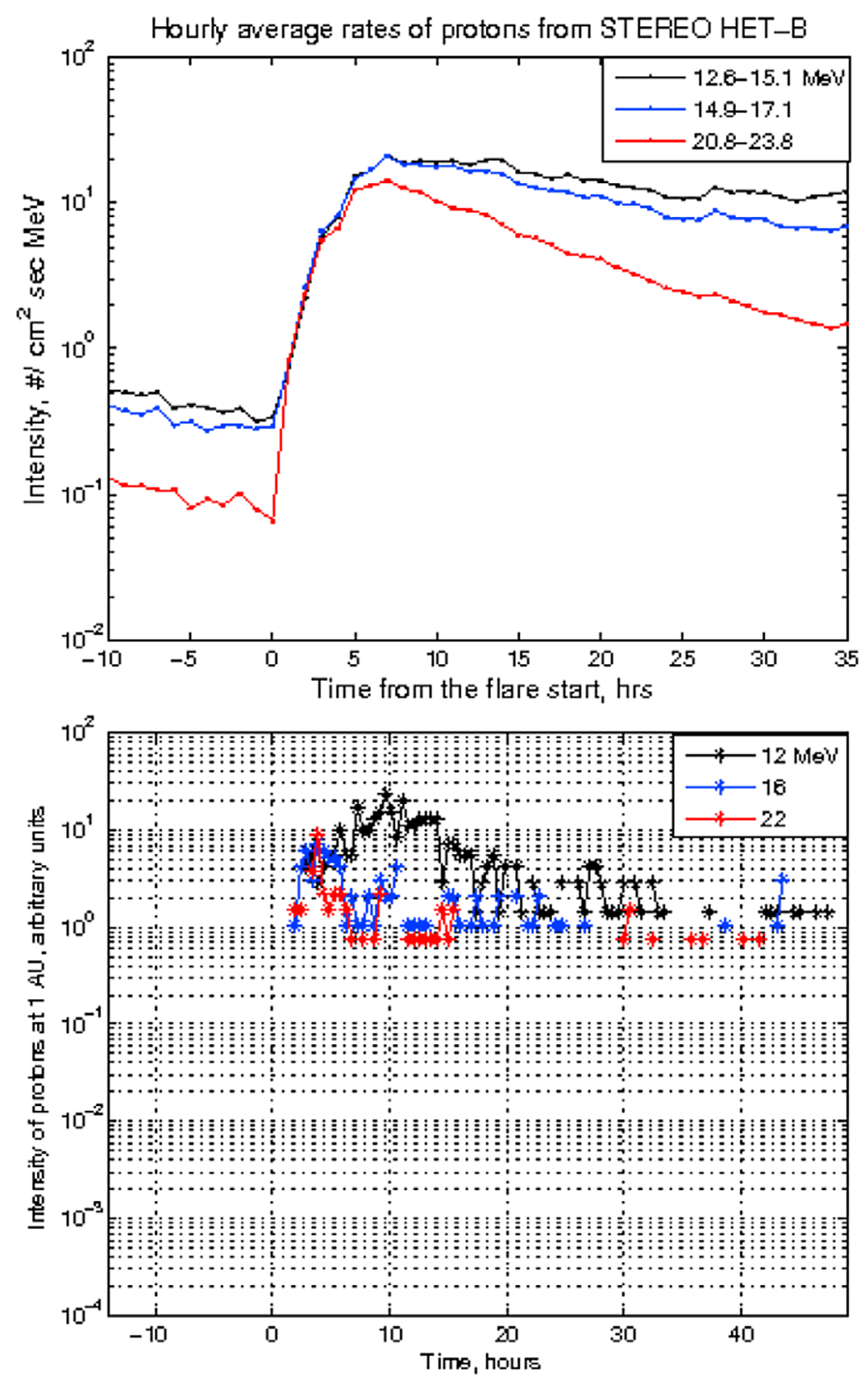

Figure 4. (top) Proton intensities derived from STEREO B-HET measurements in the high-energy range of $\sim 13-20 \mathrm{MeV}$ at $1 \mathrm{AU}$. (bottom) Corresponding modeling results in similar energy ranges with the PATH code. Note that observed shock arrival time is estimated at $\sim 35 \mathrm{~h}$ and the modeled value is $\sim 49 \mathrm{~h}$ (see Table 1). The flare start and shock arrival on both panels are at zero time and at the right-hand end of the panel, correspondingly.

flare onset time than the high-energy proton intensities. Lowenergy protons have a pronounced maximum around the shock arrival time (the ESP event) (Figure 5, top). The distinct temporal evolution of the high- and low-energy components of energetic particle intensities does not contradict the general scenario outlined above for a mixed SEP event. We interpret these results to indicate that the high-energy part of the particle population has its origin either in the flare particles or in seed particles accelerated at strong shock close to the beginning of the SEP event. This would explain the sharp rise of energetic particle intensities during the first hours of the event and the subsequent decay in intensity. As the event progresses, a portion of flare particles is absorbed by the shock and contributes to the seed population. As the shock weakens it absorbs and accelerates particles at lower energies, which causes a rise of particle intensities in low-energy channels. When the shock complex eventually reaches $1 \mathrm{AU}$ it carries with it a population of trapped particles in its environment, leading to an increase in particle intensity that is associated with an ESP event. However, we should note that the picture is typically more complex and the details of timeintensity profiles are influenced by flare location (magnetic 

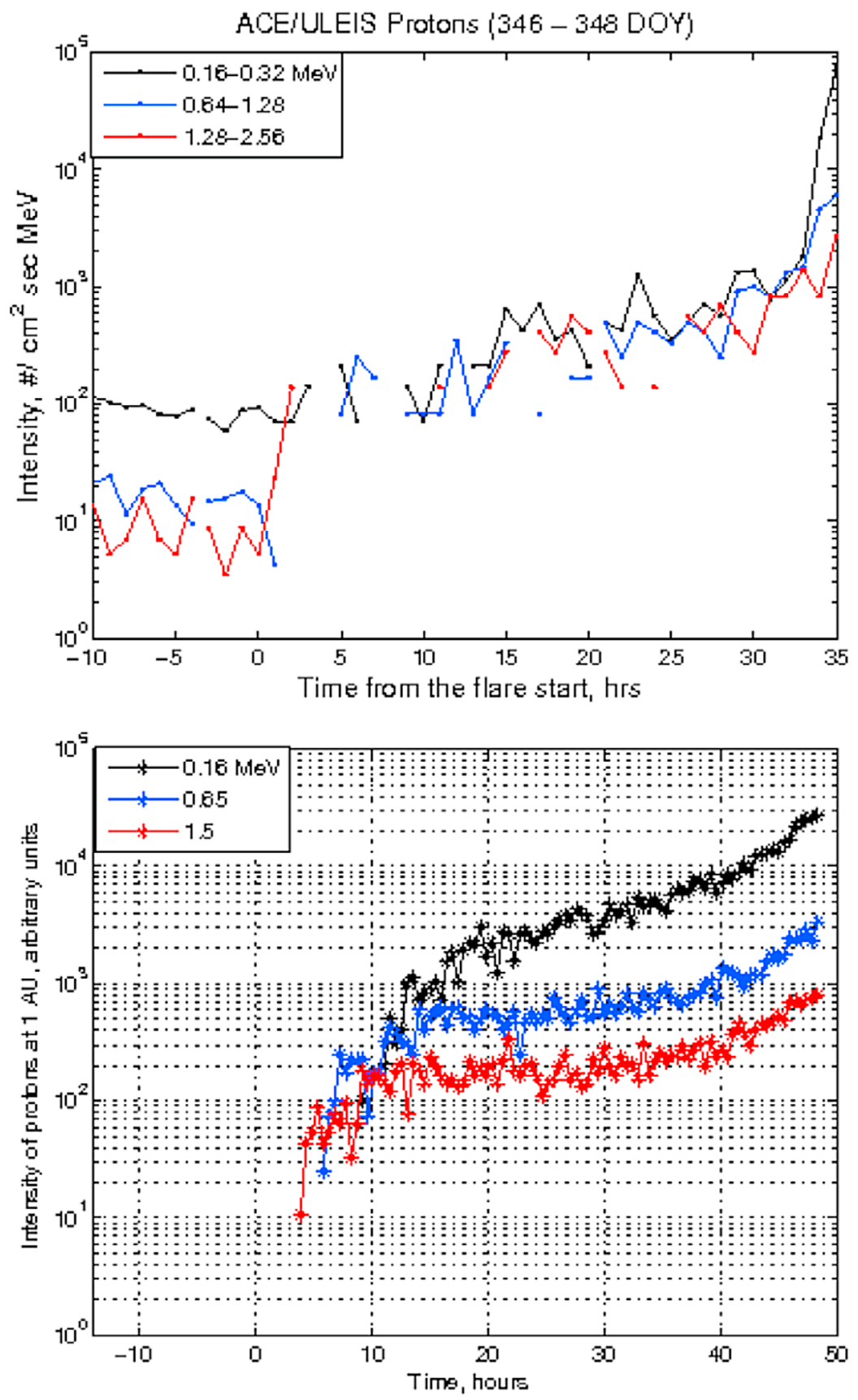

Figure 5. (top) Proton intensities derived from ACE/ULEIS measurements in a low-energy range of $\sim 160 \mathrm{keV}-3 \mathrm{MeV}$ at $1 \mathrm{AU}$. (bottom) Corresponding modeling results in similar energy ranges with the PATH code. The flare start and shock arrival on both panels are at zero time and at the right-hand end of the panel, correspondingly.

connectivity to the Earth), fractional amount and spectra of the flare particles and the shock strength. Particles accelerated at a very strong shock can contribute substantially to a medium-energy part of the particle spectrum (see section 4.2).

[16] Figure 6 (top) shows oxygen $O$ intensities in selected energy channels from the SIS instrument [Stone et al., 1998]. Note the peak at all energies shortly after the flare onset. Observations by the ULEIS instrument (Figure 7, top) show a different evolution for the time-intensity profiles of oxygen ions (at $\sim 160 \mathrm{keV} / \mathrm{nuc}-1.2 \mathrm{MeV} / \mathrm{nuc}$ ). In this case, there is a minor peak after the flare onset and a major peak at the time of shock passage for energies below $1 \mathrm{MeV}$.

\section{PATH Modeling Results and Comparison With the Observations at $1 \mathrm{AU}$}

\subsection{Shock Parameters}

[17] The main MHD shock parameters at $1 \mathrm{AU}$ were defined by using the MHD Rankine-Hugoniot conditions 

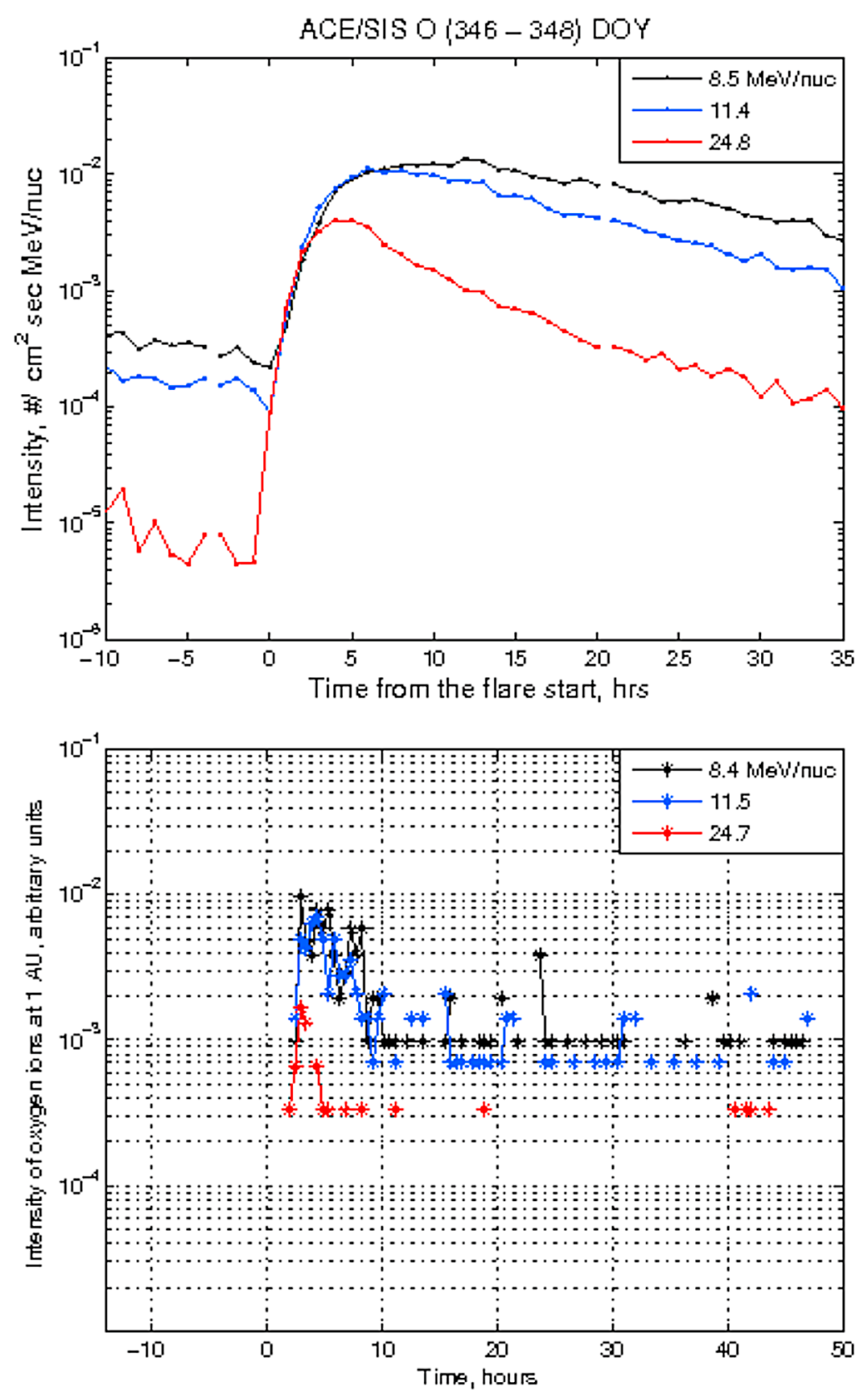

Figure 6. (top) Oxygen ion intensities derived from ACE/SIS measurements in the high-energy range of $\sim 8-25 \mathrm{MeV} /$ nuc at $1 \mathrm{AU}$. (bottom) Corresponding modeling results with the PATH code. Note the initial increase in particle intensities shortly after the flare onset time. The flare start and shock arrival on both panels are at zero time and at the right-hand end of the panel, correspondingly.

through a least squares fitting procedure [Szabo, 1994; Viñas and Scudder, 1986]. The shock angle at $1 \mathrm{AU}$ is $\sim 30^{\circ}$. In our model, we assumed spherical symmetry and studied the propagation of a 1D shock only. It should be noted that the current model includes shock launch at $\sim 20$ solar radii (or $0.1 \mathrm{AU})$. In future, this code might be linked with more advanced models of a CME shock that starts much closer to the Sun [Tsurutani et al., 2003; Zhang and Wu, 2009], thus providing a more accurate account of the early stages of an SEP event. By adjusting boundary conditions at the inner modeling boundary near the Sun, i.e., solar wind number density, kinetic temperature, velocity and interplanetary magnetic field components, we achieved a reasonable fit to the corresponding parameters observed with ACE at Earth orbit. Results are summarized in Table 1. The observed shock arrival time differs from the modeling result. This discrepancy may be explained by our simplified 1D model of shock propagation. According to the SOHO/LASCO CME Catalog (courtesy of the CDAW Data Center, GSFC) the halo CME had a linear velocity of $1774 \mathrm{~km} / \mathrm{sec}$ when first observed and a velocity of $1573 \mathrm{~km} / \mathrm{sec}$ at 20 solar radii showing a considerable decrease. This result was used as the boundary 

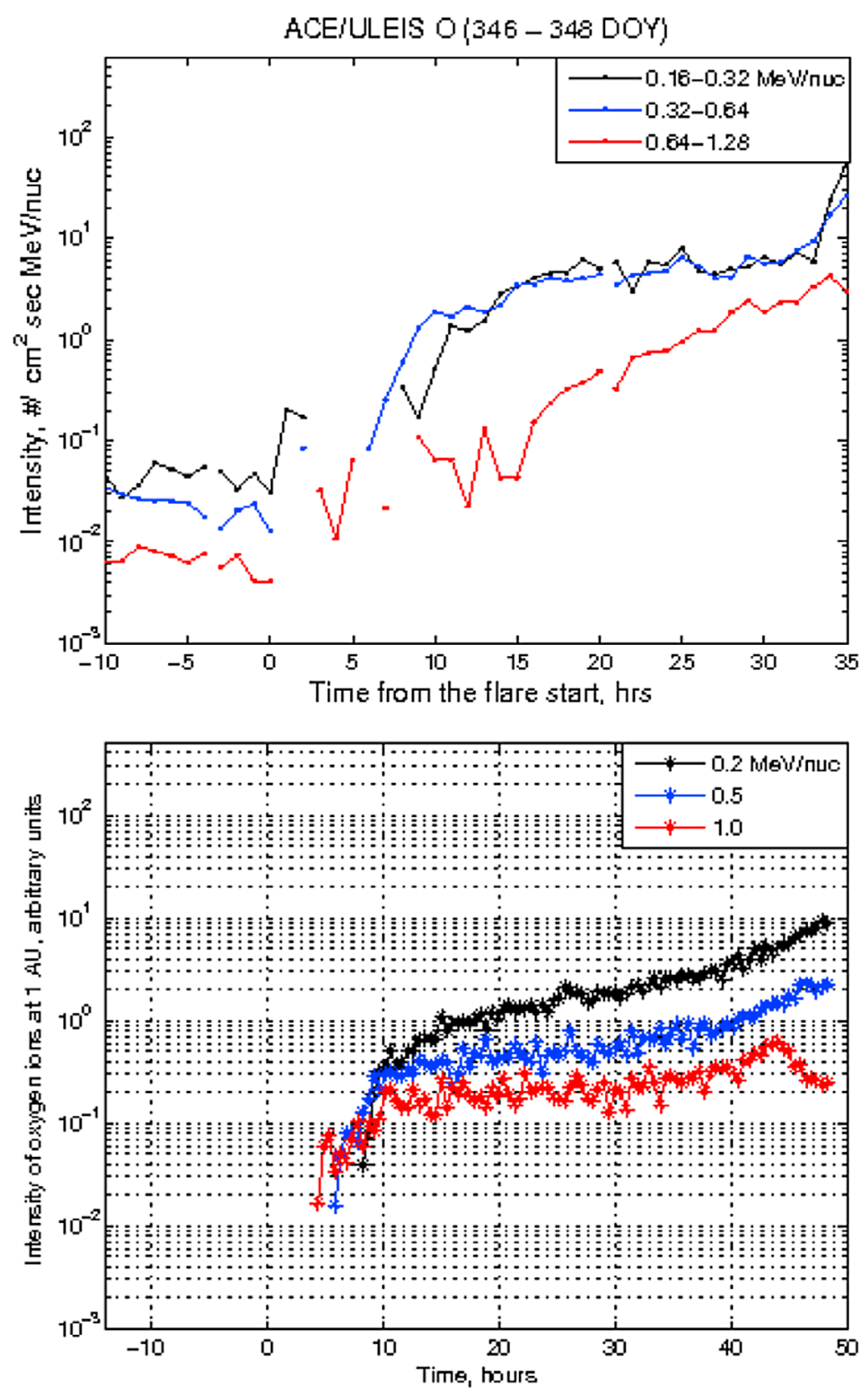

Figure 7. (top) Oxygen ion intensities derived from ACE/ULEIS measurements in a low-energy range of $\sim 160 \mathrm{keV} /$ nuc-1 MeV/nuc at $1 \mathrm{AU}$. (bottom) Corresponding modeling results with the PATH code. Notice a rise in particle intensities around the shock arrival time. The flare start and shock arrival on both panels are at zero time and at the right-hand end of the panel, correspondingly.

Table 1. Shock Parameters at $1 \mathrm{AU}^{\mathrm{a}}$

\begin{tabular}{lcccc}
\hline & $\begin{array}{c}\text { Compression } \\
\text { Ratio }\end{array}$ & $\begin{array}{c}\text { Upstream Solar } \\
\text { Wind Velocity } \\
(\mathrm{km} / \mathrm{s})\end{array}$ & $\begin{array}{c}\text { Shock Speed } \\
(\mathrm{km} / \mathrm{s})\end{array}$ & $\begin{array}{c}\text { Shock Arrival } \\
\text { Time at 1 AU } \\
(\text { hours })\end{array}$ \\
\hline Derived From ACE Observations & $3.0 \pm 0.3$ & $650 \pm 30$ & $800 \pm 50$ & $\sim 35$ \\
Modeling Result & 2.8 & 650 & 730 & $\sim 49$ \\
\hline
\end{tabular}

${ }^{\mathrm{a}}$ Modeling results using the PATH model are compared with ACE observations at 1 AU. 
condition for the CME-driven shock speed. As the shock propagates away from the Sun, in our model, it slows down and weakens [Zank et al., 2007]. The modeled shock decelerated fast at the beginning with its speed decreasing to $\sim 880 \mathrm{~km} / \mathrm{sec}$ at $0.15 \mathrm{AU}$ and then gradually to $\sim 730 \mathrm{~km} / \mathrm{sec}$ at 1 AU. Our MHD shock modeling describes the shock deceleration and we believe provides a reasonable physical description of the shock evolution up to $1 \mathrm{AU}$ where the shock speed and compression ratio are close to those observed. The deceleration history of the CME shock is automatically embedded in the model. Thus, we expect out modeling to accurately represent particle acceleration at the SEP event at a somewhat shifted time scale. However, the exact time profiles of the modeled and actual shocks may not be the same. Indeed, the modeled shock arrival time is $14 \mathrm{~h}$ longer than that observed. Therefore, the modeled time intensity profile of SEPs is also different from the observed one and it must be scaled to be compared with the observation.

[18] We understand that the picture of a shock with a smooth fixed shape is an idealized one. Possible consequences of "ripples" on a shock surface on local particle acceleration need to be considered [Li and Zank, 2006]. In any event, our modeling represents an attempt to reconstruct a general physical picture of the mixed SEP event. To summarize, the PATH code accounts for radial (temporal) changes in the shock speed and compression ratio [Zank et al., 2000, 2007] and keeps the shock geometry (obliquity) constant throughout the simulations.

\subsection{Particle Spectra at $1 \mathrm{AU}$}

[19] The seed particle population injected into the shock consists of particles of flare origin and particles of solar wind origin. The modeling was done assuming that $25 \%$ of the total number of particles in the simulation are flare particles and the rest are solar wind particles. The number is arbitrary, since we do not know at this point how to determine the ratio from observations. In actual events we might expect this ratio to change throughout the flare event according to the flare intensity profile. This is currently beyond the scope of our model. We will discuss effects of the ratio value on the event-integrated spectrum in section 5. However, the assumption of $25 \%$ is reasonable and provides a good agreement with observations (see sections 4.2 and 4.3 below). The flare spectrum is taken to be a power law distribution, $\sim p^{-4.9}$, up to a maximum particle energy of $1 \mathrm{GeV}$. This power law was chosen to comply with the observed power law spectrum of $\sim \mathrm{E}^{-2.45}$ for high-energy protons observed by SAMPEX (Figure 3). The assumed flare duration in the model was the same as that of observed (of $\sim 43$ min duration) and we do not introduce new flare particles into the system after the flare event ends. Since a numerical shock is launched in our model at $0.1 \mathrm{AU}$, we need to introduce a numerical delay between the flare onset and the start of particle acceleration at the modeled shock. This delay was chosen to be $40 \mathrm{~min}$ and was estimated from the linear speed of the CME at onset (from the SOHO/LASCO CME Catalog, courtesy of the CDAW Data Center, GSFC). Solar wind particles were injected into the shock at a minimum energy of $10 \mathrm{keV}$ and with an injection efficiency of $10 \%$ relative to the background solar wind (see Zank et al. [2000, 2007] for details). We assume that particles injected into the shock have a delta function distribution in energy.

[20] Zank et al. [2000] estimated the maximum energy to which a shock can accelerate particles locally by equating the shock dynamical time scale to the acceleration time scale. Here the dynamical time scale is defined by $\left(\mathrm{R}(\mathrm{t})-\mathrm{R}_{0}\right) /$ $(\partial \mathrm{R} / \partial \mathrm{t})$, where $R(t)$ is the shock speed in the spacecraft frame, $R_{0}$ the position of shock formation (at $\sim 5$ solar radii), and $\partial \mathrm{R} / \partial \mathrm{t}$ describes the shock speed change over time. Thus, the shock dynamic time scale is the time scale over which the shock changes significantly. The maximum accelerated energy decreases as the shock propagates from the Sun. Following their approach, we estimated maximum achievable energies for the shock-accelerated particles (near the Sun) in our modeling as approximately $34 \mathrm{MeV}, 20 \mathrm{MeV} /$ nuc and $15 \mathrm{MeV} /$ nuc, for protons, oxygen and iron ions, correspondingly. These energies decrease to $\sim 2 \mathrm{MeV}, 840 \mathrm{keV} / \mathrm{nuc}$ and $700 \mathrm{keV} /$ nuc at $1 \mathrm{AU}$, correspondingly. The estimates give us approximate upper limits in energy below which the injected solar wind particles contribute the most to the observed particle intensities. We should note that several approximations were made to derive the analytical formula. For instance, a Bohm diffusion coefficient was assumed (see Zank et al. [2000] for details). Our modeling utilizes a more realistic diffusion coefficient which is lower than the Bohm limit and might give a somewhat higher achievable maximum energy.

[21] Figure 8 (top) presents our modeled event-integrated proton spectrum superposed on the observational results shown separately in Figure 3. The simulation results show a double-power law and agree reasonably well with in situ observations. Note that the spectrum break seen in observations is not represented very accurately in our modeling. Recently, Li et al. [2009] discussed the nature of this spectral break. They related this break in the energy spectrum and its $\mathrm{Q} / \mathrm{A}$ (charge-to-mass) dependence with the shock geometry when the shock was still close to the Sun. However, only particles from the CME shock are modeled in [Li et al., 2009], but here we include particles from both the flare and the CME shock. Note that a change (increase) in the injected flare to solar wind particles ratio would contribute to (hardening of) the high-energy part of the spectrum without changing the low-energy part, while the low-energy spectrum is controlled solely by the solar wind particles (see section 5 for detailed discussion).

[22] The PATH code can describe the acceleration and transport of heavy ion species as well as protons. In our simulations we used the following charge states for the heavy ions in this SEP event: an iron charge state of 16 and an oxygen charge state of 6 (A. Labrador, personal communication, 2008). Figure 8 (middle and bottom) shows the event-integrated spectra of oxygen and iron ions. The model spectrum is superposed on event-integrated spectrum derived from ACE and SIS measurements. The plot shows good agreement of the modeling results with observations except for the lower energy portion where the observations flatten significantly.

\subsection{Time-Intensity Profiles at $1 \mathrm{AU}$}

[23] Consider now the intensity-time profiles of energetic particles at 1 AU. Figure 4 (bottom) presents simulation results that can be compared with observations made by STEREO B-HET in similar energy channels (Figure 4, top) 
for protons in the range $\geq 12 \mathrm{MeV}$. As above, we assume that $25 \%$ of the injected (or seed) population are flare particles and the rest are of the solar wind origin. The flare onset is clearly seen as a steep increase in particle intensities on both
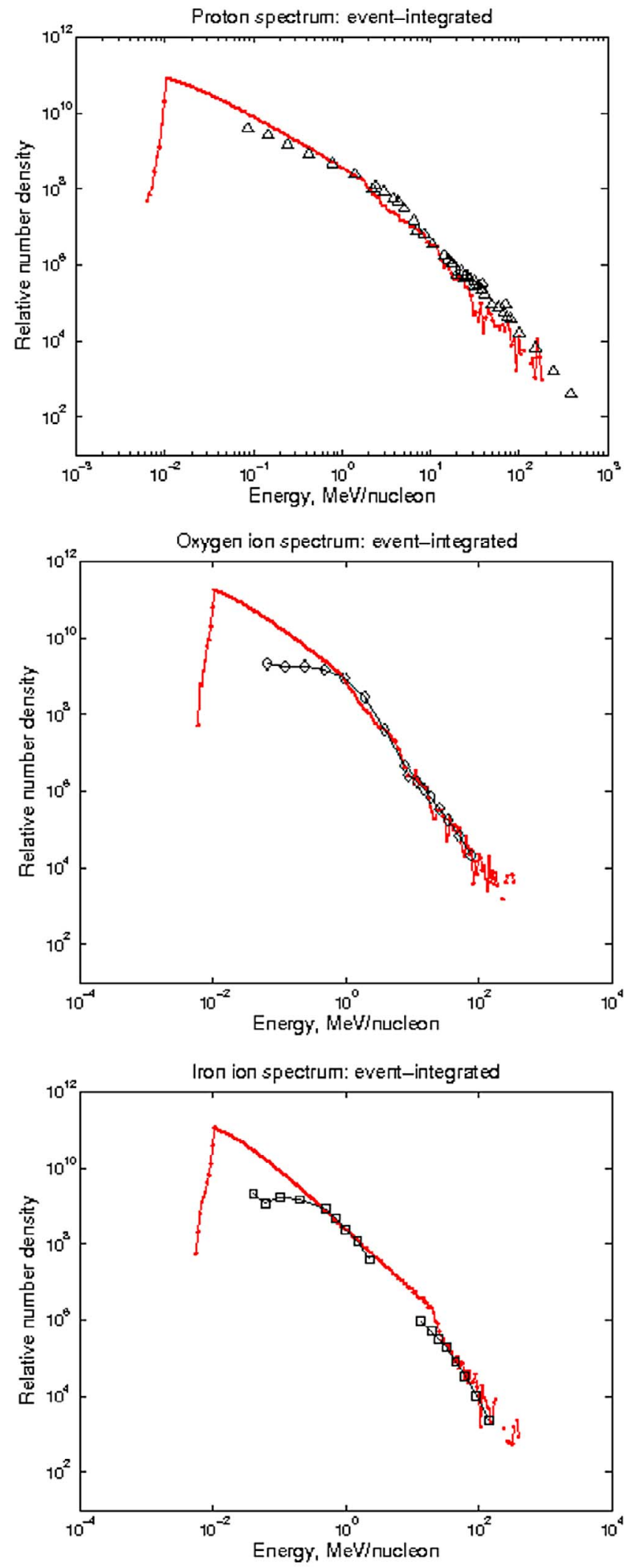

panels. A small increase around the shock arrival time at $1 \mathrm{AU}$ (see also Figure 1 by Cane et al. [1988] for a western flare when an accompanying shock is observed at Earth) is seen in the observational results at energies below $\sim 17 \mathrm{MeV}$. Note that observed shock arrival time is estimated as less than $35 \mathrm{~h}$ (see Figure 4, top) and the modeled value is $\sim 49 \mathrm{~h}$ (see Figure 4, bottom, and Table 1). A time scaling is introduced in the Figures $4-8$ to help compare observations and modeling results. A temporal dependence of the high-energy intensities is caused by transport effects. Particles scatter along magnetic field lines with the characteristic mean free path dependent on heliocentric distance and particle momentum. For about the same momentum, the mean free path increases with the heliocentric distance, and the time between two consecutive scatterings increases. Thus, particles at larger heliocentric distances tend to escape that region faster than particles closer to the Sun, causing a decrease in particle intensities with heliocentric distance. Overall, our modeling represents the general trend quite well.

[24] Simulation results for lower-energy protons at $1 \mathrm{AU}$ are shown in Figure 5, bottom. Figure 5 (top) presents observations made by ACE-ULEIS. Our simulations show that proton intensities for $\sim 1.5 \mathrm{MeV}$ particles start rising several hours after the flare onset. This occurs for lower energy particles as well, down to $\sim 160 \mathrm{keV}$. The modeled particle intensities reach maximum by the modeled shock arrival time (around $49 \mathrm{~h}$ ), which is seen by ACE (at $\sim 35 \mathrm{~h}$ ).

[25] Similar dynamics is shown in modeled time-intensity profiles for low- and high-energy oxygen ions. High-energy range particles peak around the flare onset time (Figure 6, bottom). It should be noted, however, that the counting rate of high-energy oxygen ions ( $\sim 24 \mathrm{MeV} /$ nuc after $\sim 10 \mathrm{~h}$ from the flare onset) is very low and for the purpose of comparison with observation, can be regarded as zero. Modeled lowenergy range intensities of oxygen ions (at energies below $1 \mathrm{MeV} /$ nuc) reach maximum at the end of the SEP event (Figure 7, bottom). In general the variability revealed in our simulations is consistent with the observed variability of the intensity profiles for different energy ranges (see Figure 6, top and Figure 7, top).

\section{Discussion and Conclusion}

[26] A large SEP event increases a radiation hazard at Earth orbit and can even affect (possibly cause a loss of) communication satellites [Feynman and Gabriel, 2000; Tsurutani et al., 2009]. Modeling large SEP events is important for understanding the SEP phenomenon itself and can aid in predicting the radiation environment at $1 \mathrm{AU}$ during solar events.

[27] The modeling of several SEP events, including the 13 December 2006 event, was recently undertaken by the

Figure 8. (top) Event-integrated proton spectrum. PATH code results are shown by the red line. Fluences obtained with ACE, STEREO, GOES-11 and SAMPEX are shown by triangles. (middle) Event-integrated oxygen ion spectrum. PATH code results are shown by the red line. Fluences derived from ACE measurements are shown by diamonds. (bottom) Event-integrated iron ion spectrum. PATH code results are shown by the red line. Fluences derived from ACE measurements are shown by squares. 


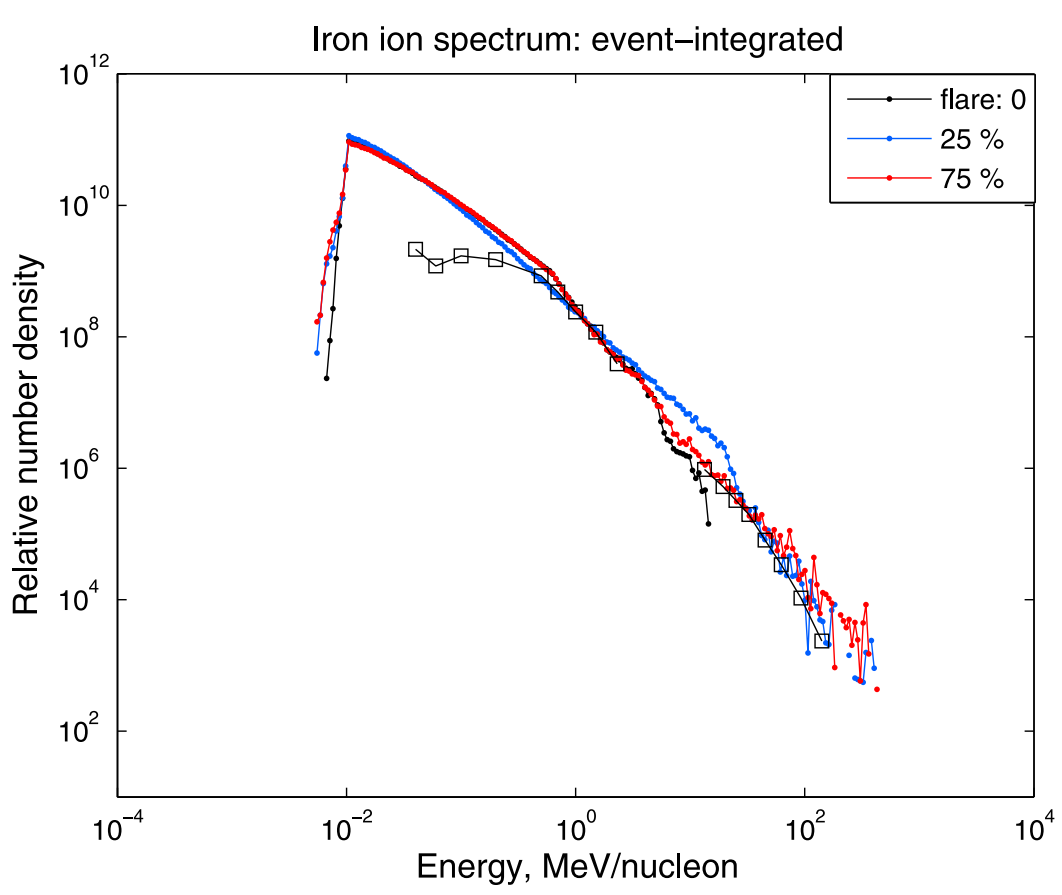

Figure 9. Event-integrated iron ion spectrum shown for different flare particle contributions to the seed population for the shock angle of $30^{\circ}$. Fluences derived from ACE measurements are shown by squares.

Center for Integrated Space weather Modeling (CISM) group [Luhmann et al., 2010]. Their numerical approach incorporated a detailed description of CME generation and evolution of related shocks [Luhmann et al., 2007]. Their results were generally consistent with observations at $1 \mathrm{AU}$ for this event. The main idea underlying the modeling of Luhmann et al. is that the influence of the shock dominates diffusive transport in determining time-intensity profiles in SEP. We certainly agree that a realistic description of CME-driven shock(s) can substantially improve the modeling of an SEP event, especially for a complex event associated with multiple shocks. However, we regard the different seed population (flare and solar wind origin) as very important for modeling SEP events, especially those originating on the western limb. Furthermore, we find transport effects also very important to explain the event spectra and the time-intensity profiles.

[28] Our modeling results show a good agreement with multisatellite observations made during the 13 December 2006 SEP event at 1AU. By selecting initial boundary conditions for our 1D MHD modeling, we matched the observed shock speed and compression ratio at $1 \mathrm{AU}$ (Table 1). The PATH code has incorporated both proton and heavy ion acceleration at an oblique and evolving shock. To our knowledge this is the first time that a reasonable agreement with observations of a specific mixed SEP event has been achieved for both particle intensity-time profiles and accelerated particle spectra, for protons and heavy ions.

[29] The low-energy part of the particle spectrum is dominated by shock accelerated particles that originated as solar wind suprathermals. The lowest energies peak when the shock arrives at 1 AU, so creating an ESP event, thus reflecting both the contribution from particles accelerated currently and those accelerated earlier and subsequently trapped in the shock complex (Figures 5 and 7). Since the shock was faster and stronger close to the Sun where the magnetic field is larger, higher energy particles are accelerated earlier. This effect is seen in the initial rise of more energetic particles followed by rises in less energetic particles (Figure 4, bottom and Figure 6, bottom). Our particle spectra reflect the general features of the observations.

[30] The flare particles discussed in the manuscript refer to particles accelerated at the flare site. These particles often have a higher $\mathrm{Fe} / \mathrm{O}$ ratio comparing to the solar wind and that of the gradual SEP events. Furthermore, flare particles are enriched in ${ }^{3} \mathrm{He}$. Since this paper does not focus on the study of composition, we list the following relevant flare material characteristics. (1) The flare spectrum is taken to be a power law distribution, $\sim p^{-4.9}$, up to a maximum particle energy of $1 \mathrm{GeV}$. (2) Flare particles are injected into the simulation system only during the 43 min of the actual flare. (3) After that time there is no influx of the flare particles into the simulation system. The remaining particles of the flare origin are subject to interaction with the shock and transport. (4) The total number of the flare particles integrated over the spectrum is 0.25 of the number of solar wind particles injected into the shock during the entire shock propagation.

[31] We assume that the high-energy part of the SEP particle spectrum in the case of a small-obliquity shock $\left(\sim 30^{\circ}\right)$ is largely determined by flare particles. If the flare contribution is absent, the high-energy part of the spectrum with particle energies above the estimated maximum values for the shockaccelerated particles disappears (not shown here). The PATH code accounts for the additional diffusive acceleration of flare particles by the shock. Our modeling results show good agreement with observed proton, oxygen and iron ion spectra (Figure 8). However, more theoretical effort (particularly in the particle transport model) is needed to adequately capture the spectral break when both flare particles and shock 
particles are present (Figure 8, top). The flare particles peak at the beginning of the SEP event, which is shortly after the onset of the flare (Figures 4 and 6). These results reflect our original assumption about the physical mechanisms underlying mixed SEP events [Cane et al., 2003] extended to include the possibility of flare particles experiencing reprocessing by a shock [Li and Zank, 2005].

[32] In our modeling, of the total number of particles initially injected, we assumed that $25 \%$ was composed of flare particles and the rest of solar wind particles for both protons and heavy ions. This is a free parameter of the model and finetuning of it (together with utilizing a temporal flare profile) could possibly provide an even better fit to the observed particle spectra. We note that an estimate for the ratio of flare particles to that of the background solar wind is hard to obtain since it depends crucially on the cutoff energy at the lowerenergy end of the accelerated population for both the flare population and that of the CME shock population. As a crude estimate, one can approximate that ratio as the ratio of the total energy released in typical large flare events and that of gradual SEP events. For two flare/CME events, Emslie et al. [2004] have estimated the total energy contained in the energetic flare particles to be $\sim 10^{34}\left(10^{30}\right)$ erg (if the cutoff of the lower energy is $0.1 \mathrm{MeV}(2.5 \mathrm{MeV})$. Most of that energy is in the form of energetic particles that precipitate back to the solar surface. A few percent gets out to the interplanetary space. Therefore outgoing energetic flare particles has $\sim 5^{*} 10^{28}$ to $5^{*} 10^{32}$ erg energy. In comparison, the kinetic energy of the CME is about $10^{32} \mathrm{erg}$. As shown by Mewaldt [2006] and Mewaldt et al. [2008b], typically 10\% of CME energy goes into accelerating SEPs based on a sample of 23 SEP events, therefore the energy in shock accelerated particles is around $10^{31} \mathrm{erg}$. The ratio of the energies contained in the shock-accelerated particles to that contained in the flare accelerated particles therefore can be between 0.005 and 5. In a following paper, Emslie et al. [2005] suggests that in large SEP events, it is perhaps reasonable to assume that the energy of the total flare accelerated particle and the kinetic energy of a CME are approximately equal. Then above argument will yield a ratio of the flare energetic particles to shock accelerated particles to be around $0.1-0.5$.

[33] In this paper we use an estimate of 0.25 for the ratio of flare accelerated particles to that of the shock accelerated. We believe that our assumed value reflects the general trend reasonably well. It is possible that this fractional choice depends on composition and thus could be different for protons and heavy ions, which could improve our modeling of high-energy part of the iron spectrum (Figure 8, bottom). However, in our modeling we keep this ratio the same for protons and heavy ions during the flare duration. Thus, we consider the energy dependence of $\mathrm{Fe} / \mathrm{O}$ ratio at $1 \mathrm{AU}$ mainly as a result of acceleration and transport effects (discussed below).

[34] To illustrate how the fractional amount of flare particles define an event-integrated spectrum we modeled the cases for which $0 \%, 25 \%$ and $75 \%$ of iron ions was contributed initially by flare accelerated particles. Eventintegrated spectra for the three cases are shown in Figure 9. Shock particles only create a spectrum which drops off at $\sim 10 \mathrm{MeV} /$ nuc for Fe ions (black line). For the case of $25 \%$ of flare particles the spectrum extends above $100 \mathrm{MeV} / \mathrm{nuc}$ (blue line) and fits the observations (black squares) well in both the lower-energy and the high-energy ends. The larger contribution of flare particles (75\%) results in slightly worse fit in the low-energy end of the spectrum (red line). This overestimation of the particle number is caused by a larger number of particles of the flare origin being trapped and/or reaccelerated by the shock. We also have more energetic particles in the high-energy region (above $10 \mathrm{MeV} / \mathrm{nuc}$ ) and a harder spectrum than in the previous case, reflecting the injection flare spectrum.

[35] Consider again the two possible mechanisms for mixed events, as discussed in section 1. An important characteristic of an SEP event is the (elemental) ratio of heavy element abundances to oxygen abundance. Based on observations for the 13 December 2006 event, Cohen et al. [2008a] discussed the dependence of composition ( $\mathrm{Fe} / \mathrm{O}$ ratio) on particle energy. They found that the $\mathrm{Fe} / \mathrm{O}$ ratio decreases to about 0.2 near $1 \mathrm{MeV} /$ nuc but increases at high energies $(>10 \mathrm{MeV} / \mathrm{nuc})$. This behavior might be associated either with particle acceleration at a quasiperpendicular shock [Tylka et al., 2005; Tylka and Lee, 2006] or the contribution of flare particles in a mixed event [Cane et al., 2003; Li and Zank, 2005]. We calculate the event-integrated $\mathrm{Fe} / \mathrm{O}$ ratio based on modeled event-integrated spectra for iron and oxygen ions. Here we assumed a power law flare spectrum $\sim p^{-4.9}$. We also introduce running averages using a window size of 5 consecutive data points. Figure 10 (top) shows that the ratio is close to 0.6 below $1 \mathrm{MeV} /$ nuc, then dips to about 0.3 and steadily increases with higher energies. This behavior is in general agreement with Figure 6 from Cohen et al. [2008a]. High-energy heavy ions above $10 \mathrm{MeV} / \mathrm{nuc}$ have relatively low count rates and estimates of $\mathrm{Fe} / \mathrm{O}$ for that energy range that show unphysical oscillatory behavior should be ignored. The low-energy part of the spectrum below $100 \mathrm{keV} / \mathrm{nuc}$ is overestimated in our modeling (see Figure 8) and corresponding estimates of $\mathrm{Fe} / \mathrm{O}$ show poor agreement with observations. Our simulation results (Figure 10, top) suggest that a flare to solar wind particle ratio of 0.25 (or $25 \%$ of the flare particles as discussed above) and the quasiparallel shock $\left(30^{\circ}\right)$ condition show an increase in the $\mathrm{Fe} / \mathrm{O}$ ratio with energy. We can also see an increase at above $\sim 2 \mathrm{MeV} /$ nuc on average (Figure 10, middle) if a larger flare contribution (with the ratio of 0.75 ) is assumed. Thus, our model reflects an important property of mixed SEP events, which is that injecting seed flare particles results in an iron-rich high-energy particle population. By way of comparison, we considered particle acceleration at a quasiperpendicular shock $\left(80^{\circ}\right)$ (Figure 10, bottom) and computed the $\mathrm{Fe} / \mathrm{O}$ ratio under the assumption of a $25 \%$ flare to solar wind seed particle population ratio. As illustrated in Figure 10 , bottom, the behavior of the $\mathrm{Fe} / \mathrm{O}$ ratio is quite similar to that of the quasiparallel case (Figure 10, middle). Thus, it does not appear that one can distinguish between particle acceleration at a quasiperpendicular or quasiparallel shock based on the composition ratio $\mathrm{Fe} / \mathrm{O}$ alone. These results do not necessarily imply that a quasiperpendicular shock alone would provide better agreement with observations for this event. We also modeled the proton spectrum for a shock with a constant angle of $80^{\circ}$. The resultant spectrum showed less agreement with observations in the 
middle energy range of $\sim 1-50 \mathrm{MeV}$ than our results for a quasiparallel shock.

[36] The propagation of energetic ions from the shock to $1 \mathrm{AU}$ in the PATH code is modeled by solving the full 1D
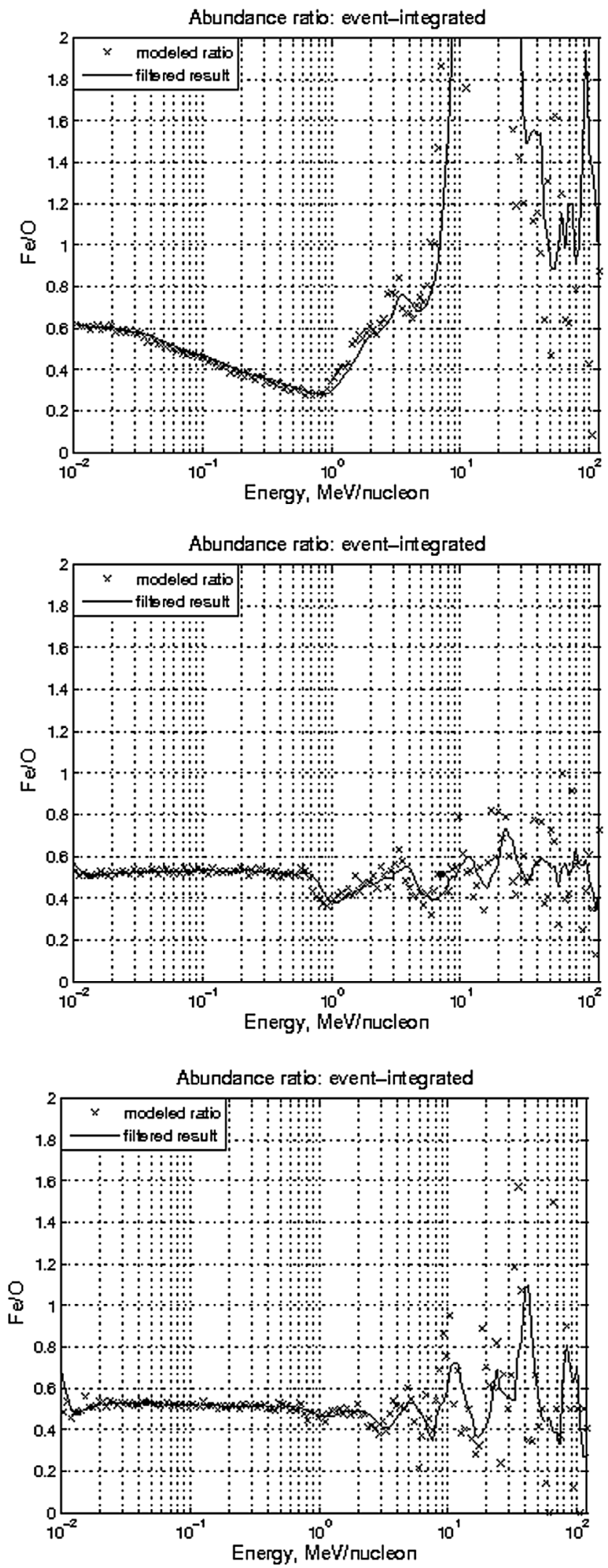

spherically symmetric Fokker-Plank equation using a Monte Carlo approach with an explicit rigidity dependence of the mean free path [Li et al., 2005]. Another general approach to particle transport is to solve the focused transport equation directly. Various methods ranging from finite difference method [Ruffolo, 1995] to Monte Carlo type test particle simulations that can be cast into stochastic differential equation method [Kocharov et al., 1998; $\mathrm{Li}, 2008$ ] have been developed. In these approaches, the rigidity dependence is through the pitch angle diffusion coefficient and it can be shown that the ratio of $\mathrm{Fe} / \mathrm{O}$ at $1 \mathrm{AU}$ is modulated by this rigidity dependence. Mason et al. [2006] have found that even though $\mathrm{Fe}$ and $\mathrm{O}$ have different charge-to-mass ratios they have virtually identical time-intensity profiles in many SEP events if one compares the $\mathrm{Fe}$ to the $\mathrm{O}$ at a somewhat higher energy/ nucleon. This suggests that propagation effects are also important in determining $\mathrm{Fe} / \mathrm{O}$.

[37] Referring to Figure 1 again, a change in the shock obliquity as the shock evolves in the solar wind can certainly be important, but the effect depends on the relative IMF connection from the shock front to the spacecraft. However, as is also apparent from Figure 1, a quasiparallel configuration is quite possible for the duration of the event, depending on the location of the spacecraft (as illustrated by spacecraft B). Nevertheless, the variation in shock obliquity from quasiperpendicular to quasiparallel (as for the spacecraft $\mathrm{A}$ ) is an important effect that needs to be included in the next version of the PATH model. As discussed by Tylka and Lee [2006], it is likely that the interplay between changing shock geometry and a composite seed particle injection source will provide even better detailed agreement between model and observations of a specific SEP event. However, as well illustrated in Figure 1, this is but one possibility of many for the way in which the shock can evolve with heliocentric distance. Not surprisingly, observations can be identified which support the importance of changing shock geometry and sometimes not. The former possibility is supported in part by observations of element abundances in the 13 December 2006 event [Cohen et al., 2008b].

[38] The extension of the PATH code to include 2D shock modeling with changing geometry will improve our estimates of the shock propagation time and allow us to better evaluate the roles and relative contributions from the above two factors for particle acceleration in a given mixed SEP event. It is quite feasible that both factors, i.e., a direct contribution from flare particles and changing shock obliquity (quasiperpendicular to quasiparallel), can provide better agreement of modeling results with the observations of some SEP events. The role of shock geometry is most likely to be manifested in the middle to high-energy part of the particle spectrum since this is where seed flare particles and particle acceleration at a quasiperpendicular shock contribute the most.

Figure 10. Abundance ratio of $\mathrm{Fe} / \mathrm{O}$ modeled for a composite particle seed population and different shock obliquities. (top) Flare particle ratio: 0.25 and the shock angle of $30^{\circ}$. (middle) Flare particle ratio: 0.75 and the shock angle of $30^{\circ}$. (bottom) Flare particle ratio: 0.25 and the shock angle of $80^{\circ}$. Symbols show modeled results. Solid curves show smoothed model results by running averages using a window size of five consecutive data points. 
[39] Acknowledgments. This work is supported in part by NASA grants NNX06AC21G, NNG04GF83G, NNG05GH38G, NNG05GM62G, and NNX07AN45G (for the EPAM and ULEIS work at APL) and NSF grants ATM0317509 and ATM0428880.

[40] Philippa Browning thanks Mathew Owens and two other reviewers for their assistance in evaluating this paper.

\section{References}

Cane, H. V., R. E. McGuire, and T. T. von Rosenvinge (1986), Two classes of solar energetic particle events associated with impulsive and long duration soft X-ray flares, Astrophys. J., 301, 448, doi:10.1086/163913.

Cane, H. V., D. V. Reames, and T. T. von Rosenvinge (1988), The role of interplanetary shocks in the longitude distribution of solar energetic particles, J. Geophys. Res., 93, 9555, doi:10.1029/JA093iA09p09555.

Cane, H. V., T. T. von Rosenvinge, C. M. S. Cohen, and R. A. Mewaldt (2003), Two components in major solar particle events, Geophys. Res. Lett., 30(12), 8017, doi:10.1029/2002GL016580.

Cane, H. V., R. A. Mewaldt, C. M. S. Cohen, and T. T. von Rosenvinge (2006), Role of flares and shocks in determining solar energetic particle abundances, J. Geophys. Res., 111, A06S90, 11,071, doi:10.1029/ 2005JA011071.

Cohen, C. M. S., R. A. Mewaldt, R. A. Leske, A. C. Cummings, E. C. Stone, M. E. Wiedenbeck, E. R. Christian, and T. T. von Rosenvinge (1999), New observations of heavy-ion-rich solar particle events from ACE, Geophys. Res. Lett., 26, 2697, doi:10.1029/1999GL900560.

Cohen, C. M. S., et al. (2008a), Comparing observations and expectations of SEP composition in the two December 2006 events, paper presented at 30th International Cosmic Ray Conference, Int. Union of Pure and Appl. Phys., Mérida, Mexico.

Cohen, C. M. S., G. M. Mason, R. A. Mewaldt, A. C. Cummings, A. W. Labrador, R. A. Leske, E. C. Stone, M. E. Wiedenbeck, and T. T. von Rosenvinge (2008b), Examination of the last large solar energetic particle events of Solar Cycle 23, AIP Conf. Proc., 1039, 118.

Desai, M. I., G. M. Mason, R. E. Gold, S. M. Krimigis, C. M. S. Cohen, R. A. Mewaldt, L. E. Mazur, and J. R. Dwyer (2006), Heavy-ion elemental abundances in large solar energetic particle events and their implications for the seed population, Astrophys. J., 649, 470, doi:10.1086/ 505649 .

Emslie, A. G., et al. (2004), Energy partition in two solar flare/CME events, J. Geophys. Res., 109, A10104, doi:10.1029/2004JA010571.

Emslie, A. G., B. R. Dennis, G. D. Holman, and H. S. Hudson (2005) Refinements to flare energy estimates: A followup to "Energy partition in two solar flare/CME events" by A. G. Emslie et al., J. Geophys. Res., 110, A11103, doi:10.1029/2005JA011305.

Feynman, J., and S. B. Gabriel (2000), On space weather consequences and predictions, J. Geophys. Res., 105, 10,543, doi:10.1029/1999JA000141.

Gordon, B. E., M. A. Lee, E. Mobius, and K. J. Trattner (1999), Coupled hydromagnetic wave excitation and ion acceleration at interplanetary traveling shocks and Earth's bow shock revisited, J. Geophys. Res., 104, 28,263, doi:10.1029/1999JA900356.

Kallenrode, M.-B. (2003), Current views on impulsive and gradual solar energetic particle events, J. Phys. G Nucl. Part. Phys., 29, 965 doi:10.1088/0954-3899/29/5/316.

Kocharov, L., R. Vainio, G. A. Kovaltsov, and J. Torsti (1998), Adiabatic deceleration of solar energetic particles as deduced from Monte-Carlo simulations of interplanetary transport, Sol. Phys., 182, 195, doi:10.1023/ A: 1005040118200 .

Lee, M. A. (1983), Coupled hydromagnetic wave excitation and ion acceleration at interplanetary traveling shocks, J. Geophys. Res., 88, 6109, doi:10.1029/JA088iA08p06109.

Li, G. (2008), On transport of charged particle in the solar wind, AIP Conf. Proc., 1039, 233

Li, G., and G. P. Zank (2005), Mixed particle acceleration at CME-driven shocks and flares, Geophys. Res. Lett., 32, L02101, doi:10.1029/ 2004GL021250.

Li, G., and G. P. Zank (2006), Particle acceleration at a rippling termination shock, AIP Conf. Proc., 858, 183.

Li, G., G. P. Zank, and W. K. M. Rice (2003), Energetic particle acceleration and transport at coronal mass ejection-driven shocks, J. Geophys Res., 108(A2), 1082, doi:10.1029/2002JA009666.

Li, G., G. P. Zank, and W. K. M. Rice (2005), Acceleration and transport of heavy ions at coronal mass ejection-driven shocks, J. Geophys. Res., 110, A06104, doi:10.1029/2004JA010600.

Li, G., G. P. Zank, O. P. Verkhoglyadova, R. A. Mewaldt, C. M. S. Cohen, G. M. Mason, and M. I. Desai (2009), Shock geometry and spectra breaks in large SEP events, Astrophys. J., 702, 998, doi:10.1088/0004$637 \mathrm{X} / 702 / 2 / 998$.
Liu, Y., J. G. Luhmann, R. Müller-Mellin, P. C. Schroeder, L. Wang, R. P. Lin, S. D. Bale, Y. Li, M. H. Acuña, and J.-A. Sauvaud (2008), A comprehensive view of the 2006 December 13 CME: From the Sun to interplanetary space, Astrophys. J., 689, 563, doi:10.1086/592031.

Luhmann, J. G., S. A. Ledvina, D. Krauss-Varban, D. Odstrcil, and P. Riley (2007), A heliospheric simulation-based approach to SEP source and transport modeling, Adv. Space Res., 40(3), 295, doi:10.1016/j. asr.2007.03.089.

Luhmann, J. G., S. A. Ledvina, D. Odstrcil, M. J. Owens, and X.-P. Zhao (2010), Liu, Yang, Riley, P., Cone model-based SEP event calculations for applications to multipoint observations, Adv. Space Res., 46, 1, doi:10.1016/j.asr.2010.03.011.

Manchester, W. B., IV, T. I. Gombosi, D. L. De Zeeuw, I. V. Sokolov, I. I. Roussev, K. G. Powell, J. Kóta, G. Tóth, and T. H. Zurbuchen (2005), Coronal mass ejection shock and sheath structures relevant to particle acceleration, Astrophys. J., 622, 1225, doi:10.1086/427768.

Manchester, W. B., IV, G. Toth, I. Sokolov, T. H. Zurbuchen, and J. Kota (2008), MHD simulations of CME-driven shocks: Structures relevant to particle acceleration, AIP Conf. Proc., 1039, 273.

Mason, G. M., et al. (1998), The Ultra-Low-Energy Isotope Spectrometer (ULEIS) for the ACE spacecraft, Space Sci. Rev., 86(1-4), 409, doi:10.1023/A:1005079930780.

Mason, G. M., M. I. Desai, C. M. S. Cohen, R. A. Mewaldt, E. C. Stone, and J. R. Dwyer (2006), The role of interplanetary scattering in Western Hemisphere large solar energetic particle events, Astrophys. J., 647(1), L65.

Matthaeus, W. H., G. Qin, J. W. Bieber, and G. P. Zank (2003), Nonlinear collisionless perpendicular diffusion of charged particles, Astrophys. J., 590, L53, doi:10.1086/376613.

Mewaldt, R. A. (2006), Solar energetic particle composition, energy spectra, and space weather, Space Sci. Rev., 124, 303, doi:10.1007/s11214006-9091-0.

Mewaldt, R. A., et al. (2008a), Observations of the December 2006 solar energetic particle events with the Low Energy Telescope (LET) on STEREO, paper presented at 30th International Cosmic Ray Conference, Int. Union of Pure and Appl. Phys., Mérida, Mexico.

Mewaldt, R. A., et al. (2008b), How efficient are coronal mass ejections at accelerating solar energetic particles?, AIP Conf. Proc., 1039, 111.

Reames, D. (1999), Particle acceleration at the Sun and in the heliosphere, Space Sci. Rev., 90(3-4), 413, doi:10.1023/A:1005105831781.

Rice, W. K. M., G. P. Zank, and G. Li (2003), Particle acceleration and coronal mass ejection driven shocks: Shocks of arbitrary strength, J. Geophys. Res., 108(A10), 1369, doi:10.1029/2002JA009756.

Ruffolo, D. (1995), Effect of adiabatic deceleration on the focused transport of solar cosmic rays, Astrophys. J., 442, 861, doi:10.1086/175489.

Sandroos, A., and R. Vainio (2007), Simulation results for heavy ion spectral variability in large gradual solar energetic particle events, Astrophys. J., 662, L127, doi:10.1086/519378.

Stone, E. C., et al. (1998), The Solar Isotope Spectrometer for the Advanced Composition Explorer, Space Sci. Rev., 86(1-4), 357, doi:10.1023/A:1005027929871

Szabo, A. (1994), An improved solution to the "Rankine-Hugoniot" problem, J. Geophys. Res., 99, 14,737, doi:10.1029/94JA00782.

Tsurutani, B. T., S. T. Wu, T. X. Zhang, and M. Dryer (2003), Coronal mass ejection (CME)-induced shock formation, propagation and some temporarily and spatially developing shock parameters relevant to particle energization, Astron. Astrophys., 412, 293.

Tsurutani, B. T., O. P. Verkhoglyadova, A. J. Mannucci, G. S. Lakhina, G. Li, and G. P. Zank (2009), A Brief Review of Solar Flare Effects on the Ionosphere, Radio Sci., 44, RSOA17, doi:10.1029/ 2008RS004029.

Tylka, A. J., and M. A. Lee (2006), A model for spectral and compositional variability at high energies in large, gradual solar particle events, Astrophys. J., 646, 1319.

Tylka, A. J., C. M. S. Cohen, W. F. Dietrich, M. A. Lee, C. G. Maclennan, R. A. Mewaldt, C. K. Ng, and D. V. Reames (2005), Shock geometry, seed populations, and the origin of variable elemental composition at high energies in large gradual solar particle events, Astrophys. J., 625 474, doi:10.1086/429384

Verkhoglyadova, O. P., G. Li, G. P. Zank, and Q. Hu (2008), Modeling a mixed SEP event with the PATH model: December 13, 2006, AIP Conf. Proc., 1039, 214

Verkhoglyadova, O. P., G. Li, G. P. Zank, Q. Hu, and R. A. Mewaldt (2009), Using the PATH code for modeling gradual SEP events in the inner heliosphere, Astrophys. J., 693, 894, doi:10.1088/0004-637X/ $693 / 1 / 894$.

Viñas, A. F., and J. D. Scudder (1986), Fast and optimal solution to the "Rankine-Hugoniot Problem", J. Geophys. Res., 91, 39, doi:10.1029/ JA091iA01p00039. 
von Rosenvinge, T. T., et al. (2008) The high energy telescope for STEREO, Space Sci. Rev., 136(1-4), 391.

von Rosenvinge, T. T., I. G. Richardson, D. V. Reames, C. M. S. Cohen, A. C. Cummings, R. A. Leske, R. A. Mewaldt, E. C. Stone, and M. E. Wiedenbeck (2009), The solar energetic particle event of 14 December 2006, Sol. Phys., 256, 443, doi:10.1007/s11207-009-9353-6.

Zank, G. P., W. K. M. Rice, and C. C. Wu (2000), Particle acceleration and coronal mass ejection driven shocks: A theoretical model, J. Geophys. Res., 105, 25,079, doi:10.1029/1999JA000455.

Zank, G. P., G. Li, V. Florinski, W. H. Matthaeus, G. M. Webb, and J. A. le Roux (2004), Perpendicular diffusion coefficient for charged particles of arbitrary energy, J. Geophys. Res., 109, A04107, doi:10.1029/ 2003JA010301.

Zank, G. P., G. Li, V. Florinski, Q. Hu, D. Lario, and C. W. Smith (2006), Particle acceleration at perpendicular shock waves: Model and observations, J. Geophys. Res., 111, A06108, doi:10.1029/2005JA011524.

Zank, G. P., G. Li, and O. P. Verkhoglyadova (2007), Particle acceleration at interplanetary shocks, Space Sci. Rev., 130, 255, doi:10.1007/s11214007-9214-2.
Zhang, T. X., and S. T. Wu (2009), Magnetohydrodynamic simulation of non-flux rope coronal mass ejections, J. Geophys. Res., 114, A05107, doi:10.1029/2008JA013860.

C. M. S. Cohen and R. A. Mewaldt, California Institute of Technology, MC 290-17, Pasadena, CA 91125-0000, USA.

D. K. Haggerty and G. M. Mason, Applied Physics Lab., Johns Hopkins University, 11100 Johns Hopkins Rd., Laurel, MD 20723-6099, USA.

Q. Hu and O. P. Verkhoglyadova, Center for Space Plasma and Aeronomic Research, University of Alabama in Huntsville, 320 Sparkman Dr., Huntsville, AL 35899-0000, USA. (olga.verkhoglyadova@jpl.nasa.gov) G. Li and G. P. Zank, Department of Physics, University of Alabama in Huntsville, UAH Technology Hall, Huntsville, AL 35899-0000, USA.

M. D. Looper, The Aerospace Corporation, POB 92957 M/S M2/260, Los Angeles, CA 90009-2957, USA.

T. T. von Rosenvinge, NASA Goddard SFC, MC 661, Bldg. 34, Rm. E216, Greenbelt, MD 20771-0001, USA. 